TRANSACTIONS OF THE

AMERICAN MATHEMATICAL SOCIETY

Volume 290, Number 1, July 1985

\title{
HARMONIC FUNCTIONS ON SEMIDIRECT EXTENSIONS OF TYPE $H$ NILPOTENT GROUPS
}

\author{
BY
}

EWA DAMEK

\begin{abstract}
Let $S=N A$ be a semidirect extension of a Heisenberg type nilpotent group $N$ by the one-parameter group of dilations, equipped with the Riemannian structure, which generalizes this of the symmetric space. Let $\left\{P_{a}(y)\right\}_{a>0}$ be a Poisson kernel on $N$ with respect to the Laplace-Beltrami operator. Then every bounded harmonic function $F$ on $S$ is a Poisson integral $F(y b)=f * P_{b}(y)$ of a function $f \in L^{\infty}(N)$. Moreover the harmonic measures $\mu_{a}^{b}$ defined by $P_{b}=P_{a} * \mu_{a}^{b}, b>a$, are radial and have smooth densities. This seems to be of interest also in the case of a symmetric space of rank 1 .
\end{abstract}

Introduction. We continue to investigate the harmonic functions on the Riemannian spaces $S$, studied in $[2, \mathbf{3}]$, with respect to the Laplace-Beltrami operator $\Delta . S$ is a semidirect product of a type $H$ nilpotent group $N$ and the one-parameter group of dilations $A$, equipped with the Riemannian structure modeled on one of the symmetric spaces of rank 1 . $S$ includes those spaces as well as many more nonsymmetric ones [3].

The formula for what should be the Poisson kernel $P_{a}, a \in A$, has been written down by J. Cygan. In [2] the author has proved that the function $P(y a)=$ $P_{a}(y), y \in N$, is harmonic, as is the function $f * P_{a}(y)$ for every $f \in L^{p}(N), 1 \leq$ $p \leq \infty$. Also it has been shown that $\lim _{a \rightarrow 0} f * P_{a}(y)=f(y)$ a.e.

The aim of this paper is to show that every bounded harmonic function on $S$ is a Poisson integral of a $L^{\infty}$ function on $N$. For the symmetric space it is, of course, well known $[6,9]$. However all the proofs we know are based on the fact that $S$ admits a large group $K$ of isometries, which leaves a point in $S$ invariant. By [3, 12] we know that such a group is in fact quite small for general $S$.

Thus our proof is based on a different idea, which seems to be new in the classical case also. The idea is based on a maximum principle and certain properties or reproducing measures $\mu_{a}^{b}$ on $N$, which are uniquely defined as solutions of the equation $P_{b}=P_{a} * \mu_{a}^{b}, b>a$. We show that these measures are radial and have smooth densities, though the explicit formulas for them, even in the case of the Siegel domain, seems to be hopeless. To investigate $\mu_{a}^{b}$ we apply a method, which began with [7] and was further developed in $[\mathbf{1}, \mathbf{1 1}]$.

The author is grateful to Andrzej Hulanicki for his ideas and helpful suggestions, which stimulated these investigations. I wish also to thank Piotr Biler, Jolanta Długosz, Paweł Głowacki and Tadeusz Pytlik for their generous assistance.

Received by the editors April 23, 1984.

1980 Mathematics Subject Classification. Primary 35J99, 43A80, 58G20; Secondary 22E25, 53C35, 43A10, 31C12.

Key words and phrases. Laplace-Beltrami operator, type $H$ nilpotent groups.

(c)1985 American Mathematical Society $0002-9947 / 85 \$ 1.00+\$ .25$ per page 
1. Preliminaries. Let $\mathcal{N}=V \oplus Z$ be a nonabelian Lie algebra of type $H$ [8] with centre $Z . V$ is the orthogonal complement to $Z$. Let $N=\exp \mathcal{N}$. We denote the element $\exp _{N}(v, z)$ by $(v, z)$ so that

$$
(v, z)\left(v^{\prime}, z^{\prime}\right)=\left(v+v^{\prime}, z+z^{\prime}+\frac{1}{2}\left[v, v^{\prime}\right]\right) .
$$

As in [2] let $S=N A$, where $A$ is the multiplicative group of $R^{+}$, be a semidirect product of $N$ and $A, A$ acting on $N$ as dilations $\delta_{a}(v, z)=\left(a v, a^{2} z\right)$. We identify $S$ with $V \times Z \times R^{+}$so that $(v, z, a)\left(v^{\prime}, z^{\prime}, a^{\prime}\right)=\left(v+a v^{\prime}, z+a^{2} z^{\prime}+\frac{1}{2} a\left[v, v^{\prime}\right], a a^{\prime}\right)$ and in the Lie algebra $S=\mathcal{N} \oplus R$ of $S$ we define the inner product

$$
\left\langle(v, z, \log a),\left(v^{\prime}, z^{\prime}, \log a^{\prime}\right)\right\rangle_{S}=\left\langle v, v^{\prime}\right\rangle+\left\langle z, z^{\prime}\right\rangle+4(\log a)\left(\log a^{\prime}\right)
$$

$[2,3]$. Let $\left\{e_{i}\right\}_{i=1, \ldots, 2 m},\left\{e_{r}\right\}_{r=2 m+1, \ldots, 2 m+l},\left\{e_{0}\right\}$, where $2 m=\operatorname{dim} V, l=$ $\operatorname{dim} Z$, be an orthonormal basis of $S$ corresponding to the decomposition $S=$ $V \oplus Z \oplus R$. Denote by $E_{\beta}$ the left-invariant vector field on $S$ determined by $e_{\beta}, \beta=0,1, \ldots, 2 m+l$. It has been shown in [2] that the Laplace-Beltrami operator associated to the left-invariant metric $\langle,\rangle_{S}$ has the form

$$
\Delta=\sum_{\beta} E_{\beta}^{2}-\frac{Q}{2} E_{0},
$$

where $Q=2 l+2 m$.

Let $\partial_{i}, \partial_{r}, \partial_{0}$ be the partial derivatives for the system of coordinates $\left(v^{i}, z^{r}, a\right)$ corresponding to $\left\{e_{i}, e_{r}, e_{0}\right\}$. Since

$$
\begin{gathered}
E_{0}=\frac{1}{2} a \partial_{0}, \quad E_{r}=a^{2} \partial_{r} \\
E_{i}=a \partial_{i}+\frac{1}{2} a \sum_{r=2 m+1}^{2 m+l}\left\langle\left[v, e_{i}\right], e_{r}\right\rangle \partial_{r}
\end{gathered}
$$

a straightforward calculation yields

$$
\begin{aligned}
\Delta= & \frac{1}{4}(1-Q) a \partial_{0}+\frac{1}{4} a^{2} \partial_{0}^{2}+a^{2}\left(a^{2}+\frac{1}{4}|v|^{2}\right) \sum_{r=2 m+1}^{2 m+l} \partial_{r}^{2} \\
& +a^{2} \sum_{i=1}^{2 m} \partial_{i}^{2}+a^{2} \sum_{r=2 m+1}^{2 m+l} \sum_{i=1}^{2 m}\left\langle\left[v, e_{i}\right], e_{r}\right\rangle \partial_{r} \partial_{i} .
\end{aligned}
$$

If $f$ is a function on $S$ depending only on $|v|, z$ and $a$, then

$$
\sum_{r=2 m+1}^{2 m+l} \sum_{i=1}^{2 m}\left\langle\left[v, e_{i}\right], e_{r}\right\rangle \partial_{r} \partial_{i} f=0 .
$$

Finally the formula for the Poisson kernel (cf. [2]) is

$$
P_{a}(y)=\frac{c a^{Q}}{\left(\left(a^{2}+|v|^{2} / 4\right)^{2}+|z|^{2}\right)^{Q / 2}}
$$

where $y=(v, z)$ and $c$ is such that $\int_{N} P_{a}(y) d y=1$.

2. A maximum principle. Let

$$
N_{a}=\{y a: y \in N\}, \quad S_{a}=\{y b: y \in N, b>a\} .
$$

We shall prove the following form of the maximum principle. 
THEOREM 2.1. For every $\varepsilon>0, M>0$ and $x=y_{1} b \in S_{a_{0}}$ there is a ball $B \subset N_{a_{0}}$ with centre in $y_{1} a_{0}$ such that, if $F$ is harmonic in $S_{a_{0}}$ (i.e. $\Delta F=0$ on $\left.S_{a_{0}}\right)$, continuous in $\bar{S}_{a_{0}}$ and $|F| \leq M$, then

$$
F(x) \leq \sup _{y \in B} F(y)+\varepsilon .
$$

PROOF. Since $\Delta$ commutes with left translations, it is sufficient to prove the theorem for $y_{1}=e=(0,0)$. Obviously, we can assume that $F \leq 0$ on $B$ and $M / \varepsilon>1$. We consider the function $G_{\varepsilon}=G_{\varepsilon}^{1}+\eta G_{\varepsilon}^{2}$, where

and

$$
G_{\varepsilon}^{1}(v, z, a)=-\varepsilon \frac{a-a_{0}}{b-a_{0}},
$$

in the domain

$$
G_{\varepsilon}^{2}(v, z, a)=-\frac{\varepsilon}{M} \log \left(1+|v|^{2}+|z|^{2}\right)
$$

$$
D=\left\{(v, z, a): a_{0}<a<\frac{b-a_{0}}{\varepsilon} M+a_{0},|v|^{2}+|z|^{2}<R^{2}\right\},
$$

where $\eta$ is sufficiently small (see below) and $R$ is such that $\varepsilon \eta \log \left(1+R^{2}\right) \geq M^{2}$. We put

Since by (1.1), (1.2)

$$
B=\left\{\left(v, z, a_{0}\right):|v|^{2}+|z|^{2} \leq R^{2}\right\}
$$

$$
\Delta G_{\varepsilon}^{1} \geq \frac{1}{4}(Q-1) \frac{\varepsilon a_{0}}{b-a_{0}}>0
$$

and $\Delta G_{\varepsilon}^{2}$ is bounded in $\left\{(v, z, a): a_{0} \leq a \leq\left(b-a_{0}\right) M / \varepsilon+a_{0}\right\}$, taking $\eta$ sufficiently small we obtain $\Delta G_{\varepsilon} \geq 0$. Moreover $G_{\varepsilon}+F \leq 0$ on $\partial D$, because $F \leq 0$ on $B$ and $F \leq M$ on the rest of the boundary. Applying the classical maximum principle for elliptic operators to $\Delta$ on $D$ we obtain $F(b) \leq \varepsilon$.

As an immediate consequence of Theorem 2.1 we have

CoROllary 2.2. For every $\varepsilon>0, M>0$ and $x=y_{1} b \in S_{a_{0}}$ there is a ball $B \subset N_{a_{0}}$ with centre in $y_{1} a_{0}$ such that if $F$ is harmonic in $S_{a_{0}}$, continuous in $\bar{S}_{a_{0}}$ and $|F| \leq M$, then

$$
|F(x)| \leq \sup _{y \in B}|F(y)|+\varepsilon .
$$

3. The representation theorem. First we are going to prove some properties of radial functions and measures on $N$. By $O(N)$ we denote the set of transformations $B: N \rightarrow N$ such that $\left.B\right|_{V}$ is orthogonal and $\left.B\right|_{Z}$ is the identity.

DEFINITION 3.1. We say that a Borel measure $\mu$ is radial if for every $B \in O(N)$ and every Borel set $X \subset N, \mu(B X)=\mu(X)$, and similarly a function $f$ is radial if $f(B y)=f(y), y \in N[\mathbf{1 1}]$.

By $M_{v}(N)$ and $L_{v}^{1}(N)$ we denote the set of radial measures and the set of radial integrable functions, respectively. In [11] F. Ricci proved that $L_{v}^{1}(N)$ is a commutative algebra and consequently since $L_{v}^{1}(N)$ is ${ }^{*}$-weakly dense in $M_{v}(N)$, so is $M_{v}(N)$.

Let $C_{0, v}(N)\left(C_{c, v}(N)\right)$ denote the set of radial continuous functions vanishing at infinity (with compact support). Since

$$
E f(y)=\int_{O(N)} f(B y) d B
$$


is a projection on the radial functions and $\langle E \mu, f\rangle=\langle\mu, E f\rangle$ is a projection on radial measures, the dual of $C_{0, v}(N)$ is $M_{v}(N)$. Of course, by (1.3), $P_{a}, a>0$, are radial.

Lemma 3.2. The set $R^{a}=\left\{f * P_{a}: f \in C_{c, v}(N)\right\}$ is dense in $C_{0, v}(N)$.

PROOF. Our proof is a modification of that of Przebinda [10]. Let $\mu \in M_{v}(N)$ be a functional vanishing on $R^{a}$, i.e. $0=\left\langle f * P_{a}, \mu\right\rangle=\left\langle f, \mu * P_{a}\right\rangle$. Thus

$$
\mu * P_{a}=0 \text {. }
$$

Now we consider the harmonic function $F(y b)=\mu * P_{b}(y), b>0$, on $S$. By (3.1) and the maximum principle $F(y b)=0$ for $b>a$. Since $F$ is harmonic, it is real analytic and so $F(y b)=0$ for all $b>0$. Thus $\mu=0$, because $P_{a}$ is an approximate identity.

Similarly we have

LEMMA 3.3. The set $T^{a}=\left\{f * P_{a}: f \in C_{c}(N)\right\}$ is dense in $C_{0}(N)$.

Now we return to our main theme. Let

$$
H^{a}=\left\{F \in C^{2}\left(S_{a}\right) \cap C\left(\bar{S}_{a}\right) \cap L^{\infty}\left(\bar{S}_{a}\right): \Delta F=0\right\}, \quad U^{a}=\left\{\left.F\right|_{N_{a}}: F \in H^{a}\right\} .
$$

In view of the maximum principle the mapping $\left.F \rightarrow F\right|_{N_{a}}$ is injective. Since $T^{a}$ is dense in $C_{0}(N)$, the maximum principle implies that for every $f$ in $C_{0}(N)$ there is an $F$ in $H^{a}$ such that $f=\left.F\right|_{N_{a}}$. For every $x \in S$ we can define the functional

$$
\left\langle\varphi_{a}^{x}, f\right\rangle=F(x), \quad f \in U^{a} \text { and } f=\left.F\right|_{N_{a}} .
$$

By Theorem 2.1 $\left\|\varphi_{a}^{x}\right\| \leq 1$. $\varphi_{a}^{x}$ defines a bounded measure $\mu_{a}^{x}$ such that

$$
F(x)=f * \mu_{a}^{x}(e), \quad f \in C_{0}(N) \text { and } f=\left.F\right|_{N_{a}} .
$$

Let us list a few elementary properties of $\mu_{a}^{x}$. We have

$$
\mu_{a}^{y x}=\mu_{a}^{x} * \delta_{y^{-1}}
$$

because for $g$ in the dense set $T^{a}$

$$
g * \mu_{a}^{y x}(e)=g * \mu_{a}^{x} * \delta_{y^{-1}}(e) .
$$

Putting $x=b$ and $g=F_{a}=\left.F\right|_{N_{a}}$ in (3.4) we obtain

$$
F(y b)=F_{a} * \mu_{a}^{b}(y) \text { for } F \in H^{a} \cap C_{0}\left(\vec{S}_{a}\right) .
$$

In particular

$$
P_{b}=P_{a} * \mu_{a}^{b}
$$

(3.6) immediately gives

$$
\left\|\mu_{a}^{b}\right\|=1 \quad \text { and } \quad \mu_{a}^{b} \geq 0,
$$

and (3.6) combined with Lemma 3.3 yields

$$
\mu_{c}^{a} * \mu_{a}^{b}=\mu_{c}^{b} .
$$

LEMMA 3.4. The measures $\mu_{a}^{b}$ are uniquely defined by (3.6).

PROOF. If $P_{a} * \nu=0$, then by Lemma $3.3 \nu$ defines the zero functional on $C_{0}$; hence $\nu=0$. 
LEMMA 3.5. If $f \in C_{0, v}(N)$, then $f * \mu_{a}^{b} \in C_{0, v}(N)$.

Proof. If $f \in R^{a}$, that is $f=g * P_{a}$ for a $g \in C_{c, v}(N)$, then by (3.6) $f * \mu_{a}^{b}=g * P_{b}$, which is radial. The assertion follows now by Lemma 3.2.

LEMMA 3.6. The measures $\mu_{a}^{b}$ are radial.

Proof. Let $f \in C_{0}(N)$ and $B \in O(N)$. By the previous lemma $P_{\varepsilon} * \mu_{a}^{b} \in L_{v}^{1}(N)$ for $\varepsilon>0$. Then we have

$$
\left\langle P_{\varepsilon} * f, \mu_{a}^{b}\right\rangle=\left\langle f, P_{\varepsilon} * \mu_{a}^{b}\right\rangle=\left\langle f \circ B, P_{\varepsilon} * \mu_{a}^{b}\right\rangle=\left\langle P_{\varepsilon} *(f \circ B), \mu_{a}^{b}\right\rangle .
$$

Hence, if $\varepsilon \rightarrow 0$, we obtain $\left\langle f, \mu_{a}^{b}\right\rangle=\left\langle f \circ B, \mu_{a}^{b}\right\rangle$, which completes the proof.

Now we look at the relation of these measures to bounded harmonic functions. First of all we notice that (3.2) is true for all $f \in U^{a}$, because the functional $\varphi_{a}^{x}$ is defined on the space $U^{a} \subset C(N) \cap L^{\infty}(N)$ and in view of (3.7) attains its norm on $C_{0}(N)$. As before, (3.2) combined with (3.3) gives

$$
F(y b)=F_{a} * \mu_{a}^{b}(y) \text { for } F \in H^{a} .
$$

Now we are in a position to prove the main theorem of this section.

THEOREM 3.7. If $F$ is a bounded harmonic function on $S$, then there is an $f \in L^{\infty}(N)$ such that $F(y b)=f * P_{b}(y)$.

PROOF. Since the family $\left\{F_{a}\right\}_{a>0}$ is uniformly bounded there is a sequence $F_{a_{n}}$ which is convergent *-weakly to a function $f \in L^{\infty}(N)$ when $a_{n} \rightarrow 0$. In particular, $F_{a_{n}} * P_{b} \rightarrow f * P_{b}$. On the other hand, Lemma 3.6 and (3.9) imply

$$
F_{a_{n}} * P_{b}=F_{a_{n}} *\left(P_{a_{n}} * \mu_{a_{n}}^{b}\right)=\left(F_{a_{n}} * \mu_{a_{n}}^{b}\right) * P_{a_{n}}=F_{b} * P_{a_{n}} \text {. }
$$

Since $P_{a_{n}}$ is an approximate identity, $F_{b} * P_{a_{n}} \rightarrow F_{b}$ a.e. when $a_{n} \rightarrow 0$ [2] and the theorem follows.

4. Smoothness of $\mu_{a}^{b}$. In this section we investigate more precisely the measures $\mu_{a}^{b}$ by means of the Gelfand transform of $L_{v}^{2}(N)$. The Gelfand transform of $L_{v}^{1}(N)$ is described in [11]. There are two families of multiplicative functionals. For a real nonnegative $\rho$ we write

$$
\hat{f}(\rho)=\int_{N} f(v, z) e^{i \rho\left\langle v, v_{0}\right\rangle} d v d z
$$

where $v_{0}$ is a fixed unit vector in $V$. For $w \in Z \backslash\{0\}$ and a nonnegative integer $n$ we have

$$
\hat{f}(w, n)=\left(\begin{array}{c}
n+m-1 \\
n
\end{array}\right)^{-1} \int_{N} f(v, z) e^{i\langle w, z\rangle} \mathcal{L}_{n}^{m-1}\left(\frac{1}{2}|w||v|^{2}\right) d z d v
$$

where $\mathcal{L}_{n}^{m-1}(r)=e^{-r / 2} L_{n}^{m-1}(r)$ and $L_{n}^{m-1}(r)$ is the Laguerre polynomial of degree $n$ and order $m-1$ [4]. Formula (4.1) defines a unitary operator

$$
\mathcal{F}: L_{v}^{2}(N) \rightarrow \not{H}=\left\{\chi:\|\chi\|_{\mathcal{H}}^{2}=\int_{Z} \sum_{n=0}^{\infty}|\chi(w, n)|^{2}\left(\begin{array}{c}
n+m-1 \\
n
\end{array}\right)|w|^{m} d w<\infty\right\} ;
$$

that is a Plancherel theorem holds [11]:

$$
\|f\|_{L^{2}}^{2}=(2 \pi)^{-m-l}\|\hat{f}\|_{\mathcal{H}}^{2} \text {. }
$$


Thus, of course, we have

$$
\mathcal{F}\left(P_{a} * f\right)=\mathcal{F} P_{a} \cdot \mathcal{F} f
$$

Let

$$
\mathcal{F}^{-1}(\chi)=(2 \pi)^{-m-l} \sum_{n=0}^{\infty} \int_{Z} \chi(w, n) e^{-i\langle w, z\rangle} \mathcal{L}_{n}^{m-1}\left(\frac{1}{2}|w||v|^{2}\right)|w|^{m} d w
$$

Applying the ordinary inversion formula for the Fourier transform and the orthogonality relation

$$
\frac{n !}{(n+m-1) !} \int_{0}^{\infty} L_{n}^{m-1}(r) L_{n_{1}}^{m-1}(r) r^{m-1} e^{-r} d r=\delta_{n, n_{1}}
$$

we obtain by routine calculation that

$$
\mathcal{F}^{-1} \circ \mathcal{F}=I \text { and } \mathcal{F} \mathcal{F}^{-1}=I .
$$

Using (4.3) we can write the formula for what should be the density of $\mu_{a}^{b}, b>a$. First we show that $\hat{P}_{b}(w, n) / \hat{P}_{a}(w, n) \in \mathcal{H}$, and second that $\psi_{a}^{b}=\mathcal{F}^{-1}\left(\hat{P}_{b} / \hat{P}_{a}\right)$ satisfies the equation $P_{b}=P_{a} * \psi_{a}^{b}$. If we also prove that $\psi_{a}^{b} \in L_{v}^{1}(N)$, then we shall have $\mu_{a}^{b}=\psi_{a}^{b}$.

\section{LEMMA 4.1 .}

$$
\begin{aligned}
\hat{P}_{a}(w, n)= & c_{1}|w|^{-m} e^{-|w| a^{2}} \\
& \times \int_{0}^{\infty}\left(\frac{t}{1+t}\right)^{n} e^{-2|w| a^{2} t} t^{(2 m+l-1) / 2}(1+t)^{(l-1) / 2} d t
\end{aligned}
$$

where

$$
c_{1}=2^{m+1} \pi^{(2 m+l+1) / 2} c a^{Q}\left(\Gamma\left(\frac{Q}{2}\right)\right)^{-1}\left(\Gamma\left(\frac{2 m+l+1}{2}\right)\right)^{-1}
$$

and $c$ is the constant in (1.3).

Proof. By (5.1) of [1] and (1.3) we have

$$
\begin{aligned}
\int_{Z} P_{a}(v, z) e^{i\langle w, z\rangle} d z= & c_{2} e^{-|w|\left(a^{2}+|v|^{2} / 4\right)} \\
& \times \int_{0}^{\infty} e^{-|w|\left(a^{2}+|v|^{2} / 4\right) t}\left(t^{2}+2 t\right)^{(2 m+l-1) / 2} d t
\end{aligned}
$$

where

$$
c_{2}=c a^{Q} 2^{1-2 m-l} \pi^{(l+1) / 2}\left(\Gamma\left(\frac{Q}{2}\right) \cdot \Gamma\left(\begin{array}{c}
2 m+l+1 \\
2
\end{array}\right)\right)^{-1} .
$$

Hence

$$
\text { (4.6) } \begin{aligned}
\hat{P}_{a}(w, n)= & \left(\begin{array}{c}
n+m-1 \\
n
\end{array}\right)^{-1} c_{2} e^{-|w| a^{2}} \int_{V} \int_{0}^{\infty} e^{-|w|\left(a^{2}+|v|^{2} / 4\right) t} \\
& \times\left(t^{2}+2 t\right)^{(2 m+l-1) / 2} e^{-|w||v|^{2} / 2} L_{n}^{m-1}\left(\frac{1}{2}|w||v|^{2}\right) d t d v
\end{aligned}
$$


Integrating first over $v$, by

$$
L_{n}^{m-1}(r)=\frac{1}{n !} r^{-m+1} e^{r} D^{n}\left(r^{n+m-1} e^{-r}\right)
$$

we obtain

$$
\begin{aligned}
& \int_{V} e^{-|w||v|^{2} t / 4} e^{-|w||v|^{2} / 2} L_{n}^{m-1}\left(\frac{1}{2}|w||v|^{2}\right) d v \\
& =\left(\frac{2}{|w|}\right)^{m} \frac{\pi^{m}}{n !(m-1) !} \int_{0}^{\infty} e^{-r t / 2} D^{n}\left(r^{n+m-1} e^{-r}\right) d r
\end{aligned}
$$

which by parts is equal to

$$
\left(\frac{2}{|w|}\right)^{m} \frac{\pi^{m}}{n !(m-1) !}\left(\frac{t}{2}\right)^{n} \frac{(n+m-1) !}{(1+t / 2)^{n+m}}
$$

Finally putting (4.7) into (4.6) we get (4.4).

LEMMA 4.2 .

$$
\frac{\hat{P}_{b}(w, n)}{\hat{P}_{a}(w, n)} \leq \begin{cases}c_{3} e^{-c_{4} \sqrt{|w| n}} e^{-|w|\left(b^{2}-a^{2}\right)} & \text { if } 0<|w| \leq 1, \\ c_{3} e^{-c_{4} \sqrt{n}} e^{-|w|\left(b^{2}-a^{2}\right)}|w|^{(l-1) / 2} & \text { if }|w|>1,\end{cases}
$$

where $c_{3}=(b / a)^{l-1}+\left(b^{2}+1\right)^{(l-1) / 2} c_{5}, c_{4}=\left(b^{2}-a^{2}\right) /\left(c_{6}+b^{2}\right), c_{6}=\frac{3}{2} a+1, c_{5}>0$ depends only on $m+l-1$ and $a$.

ProOF. By (4.4)

$$
\frac{\hat{P}_{b}(w, n)}{\hat{P}_{a}(w, n)}=e^{-|w|\left(b^{2}-a^{2}\right)} \frac{\int_{0}^{\infty} e^{-2 t}\left(t /\left(t+|w| b^{2}\right)\right)^{n} t^{(2 m+l-1) / 2}\left(|w| b^{2}+t\right)^{(l-1) / 2} d t}{\int_{0}^{\infty} e^{-2 t}\left(t /\left(t+|w| a^{2}\right)\right)^{n} t^{(2 m+l-1) / 2}\left(|w| a^{2}+t\right)^{(l-1) / 2} d t} .
$$

Let

$$
I_{n}(a, A)=\int_{0}^{A} e^{-2 t}\left(\frac{t}{t+|w| a^{2}}\right)^{n} t^{(2 m+l-1) / 2}\left(|w| a^{2}+t\right)^{(l-1) / 2} d t
$$

and

$$
J_{n}(a, A)=\int_{A}^{\infty} e^{-2 t}\left(\frac{t}{t+|w| a^{2}}\right)^{n} t^{(2 m+l-1) / 2}\left(|w| a^{2}+t\right)^{(l-1) / 2} d t .
$$

Analogously we define $I_{n}(b, A)$ and $J_{n}(b, A)$. We estimate separately

$$
\text { (i) } \frac{I_{n}\left(b, c_{6} \sqrt{n|w|}\right)}{I_{n}(a, \infty)} \text { and (ii) } \frac{J_{n}\left(b, c_{6} \sqrt{n|w|}\right)}{I_{n}(a, \infty)} \text {. }
$$

Since $\left(t+|w| a^{2}\right) /\left(t+|w| b^{2}\right)$ increases,

$$
\begin{gathered}
I_{n}\left(b, c_{6} \sqrt{n|w|}\right)=\int_{0}^{c_{6} \sqrt{n|w|}} e^{-2 t}\left(\frac{t}{t+|w| a^{2}}\right)^{n}\left(\frac{t+|w| a^{2}}{t+|w| b^{2}}\right)^{n} t^{(2 m+l-1) / 2} \\
\times\left(|w| a^{2}+t\right)^{(l-1) / 2}\left(\frac{|w| b^{2}+t}{|w| a^{2}+t}\right)^{(l-1) / 2} d t \\
d \leq\left(\frac{b}{a}\right)^{l-1}\left(\frac{c_{6} \sqrt{n}+\sqrt{|w|} a^{2}}{c_{6} \sqrt{n}+\sqrt{|w|} b^{2}}\right)^{n} I_{n}\left(a, c_{6} \sqrt{n|w|}\right) .
\end{gathered}
$$


Hence for (i) we have

$$
\frac{I_{n}\left(b, c_{6} \sqrt{n|w|}\right)}{I_{n}(a, \infty)} \leq\left(\frac{b}{a}\right)^{l-1} \exp \left(-\frac{b^{2}-a^{2}}{c_{6}+\sqrt{|w|} b^{2}} \sqrt{|w| n}\right) .
$$

To estimate (ii), we notice that if $t \geq c_{6} \sqrt{n|w|}$, then

$$
\left(|w| b^{2}+t\right) \leq \begin{cases}\left(b^{2}+1\right) t & \text { if }|w| \leq 1 \\ \left(b^{2}+1\right)|w| t & \text { if }|w|>1\end{cases}
$$

and

$$
J_{n}\left(b, c_{6} \sqrt{n|w|}\right) \leq c_{7} \int_{c_{6} \sqrt{n|w|}}^{\infty} e^{-2 t} t^{m+l-1} d t
$$

where $c_{7}=\left(b^{2}+1\right)^{(l-1) / 2} \max \left(1,|w|^{(l-1) / 2}\right)$. Since

$$
\left(\frac{t}{t+|w| a^{2}}\right)^{n} \geq\left(\frac{t}{t+|w| a^{2}}\right)^{t^{2} / a^{2}|w|} \geq e^{-t}
$$

for $t \geq a \sqrt{n|w|}$, we have

$$
I_{n}(a, \infty) \geq \int_{a \sqrt{n|w|}}^{\infty} e^{-3 t} t^{m+l-1} d t .
$$

There is a constant $c_{5}$ depending only on $m+l-1, a$ and $c_{6}$ such that

$$
\begin{aligned}
\frac{\int_{c_{6} \sqrt{n|w|}}^{\infty} e^{-2 t} t^{m+l-1} d t}{\int_{a \sqrt{n|w|}}^{\infty} e^{-3 t} t^{m+l-1} d t} & \leq c_{5} \exp \left(\left(-2 c_{6}+3 a\right) \sqrt{n|w|}\right) \\
& =c_{5} e^{-2 \sqrt{n|w|}} .
\end{aligned}
$$

Finally putting (4.10)-(4.12) together we obtain

$$
\frac{J_{n}\left(b, c_{6} \sqrt{n|w|}\right)}{I_{n}(a, \infty)} \leq c_{7} c_{5} e^{-2 \sqrt{n|w|}}
$$

which with (4.9) implies (4.8).

LEMMA 4.3. For every nonnegative integer $p$

$$
\sum_{n=0}^{\infty} \int_{Z}\left|\frac{\hat{P}_{b}(w, n)}{\hat{P}_{a}(w, n)}\right|^{2}(2 n+m)^{p} n^{m-1}|w|^{m+p} d w<\infty .
$$

ProOF. Let $h_{n}(w)=\left|\hat{P}_{b}(w, n) / \hat{P}_{a}(w, n)\right|^{2}|w|^{m+p}$. Since

$$
\int_{|w| \geq 1} h_{n}(w) d w \leq c_{3}^{2} e^{-2 c_{4} \sqrt{n}} \int_{|w| \geq 1} \exp \left(-2|w|\left(b^{2}-a^{2}\right)\right)|w|^{m+p+l-1} d w
$$

and

$$
\sum_{n=0}^{\infty} e^{-2 c_{4} \sqrt{n}}(2 n+m)^{p} n^{m-1}<\infty
$$

by (4.8), we have

$$
\sum_{n=0}^{\infty}(2 n+m)^{p} n^{m-1} \int_{|w| \geq 1} h_{n}(w) d w<\infty
$$


On the other hand,

$$
\begin{aligned}
\int_{|w| \leq 1} h_{n}(w) d w & \leq c_{3}^{2} \int_{|w| \leq 1} \exp \left(-2 c_{4} \sqrt{n|w|}\right) \exp \left(-2|w|\left(b^{2}-a^{2}\right)\right)|w|^{m+p} d w \\
& \leq c_{3}^{2} \int_{Z} e^{-2 c_{4} \sqrt{n|w|}}|w|^{m+p} d w \\
& =c_{3}^{2} \frac{2 l \pi^{1 / 2}}{\Gamma(l / 2+1)} \frac{(2(m+p+l)-1) !}{\left(2 c_{4}\right)^{2(m+p+l)}} \frac{1}{n^{m+p+l}}
\end{aligned}
$$

and $\sum_{n=0}^{\infty}(2 n+m)^{p} / n^{1+p+l}<\infty$ for $l>0$. Hence also

$$
\sum_{n=0}^{\infty}(2 n+m)^{p} n^{m-1} \int_{|w| \leq 1} h_{n}(w) d w<\infty
$$

which concludes the proof.

THEOREM 4.4. The measures $\mu_{a}^{b}$ are absolutely continuous and their densities $\psi_{a}^{b}$ are given by the formula

$$
\psi_{a}^{b}(v, z)=(2 \pi)^{-l-m} \sum_{n=0}^{\infty} \int_{Z} \frac{\hat{P}_{b}(w, n)}{\hat{P}_{a}(w, n)} \mathcal{L}_{n}^{m-1}\left(\frac{1}{2}|w||v|^{2}\right) e^{-i\langle w, z\rangle}|w|^{m} d w .
$$

PROOF. The previous lemma and the inequality

$$
\left(\begin{array}{c}
n+m-1 \\
n
\end{array}\right) \leq \frac{\text { const }}{(m-1) !} n^{m-1}
$$

yield $\hat{P}_{b} / \hat{P}_{a} \in \mathcal{H}$. Hence $\psi_{a}^{b} \in L_{v}^{2}(N), \mathcal{F}\left(\psi_{a}^{b}\right)=\hat{P}_{b} / \hat{P}_{a}$ and by $(4.2) \mathcal{F}\left(P_{b}\right)=$ $\mathcal{F}\left(P_{a} * \psi_{a}^{b}\right)$. Thus we have

$$
P_{b}=P_{a} * \psi_{a}^{b}
$$

Let $f$ be a continuous function with compact support. Then by $(4.14)\left(f * P_{a}\right) *$ $\psi_{a}^{b}(e)=\left(f * P_{a}\right) * \mu_{a}^{b}(e)$, which shows that $\psi_{a}^{b}$ defines a continuous functional on $C_{0}(N)$. Hence $\psi_{a}^{b} \in L^{1}(N)$ and in view of the uniqueness of $\mu_{a}^{b}$ as the solution of (3.6) $\psi_{a}^{b}$ is the density of $\mu_{a}^{b}$.

THEOREM 4.5. The functions $\psi_{a}^{b}$ are smooth and, for every left-invariant operator $\partial$ on $N, \partial\left(\psi_{a}^{b}\right) \in L^{2}(N)$.

Proof. Let $L=-\sum_{i=1}^{2 m} E_{i}^{2}$. Here $E_{i}$ is the left-invariant field on $N$ corresponding to $e_{i}$. Analogously to the case of $\Delta$ we can easily check that

$$
L=\sum_{i=1}^{2 m} \partial_{i}^{2}+\sum_{s=2 m+1}^{2 m+l} \sum_{i=1}^{2 m}\left\langle\left[v, e_{i}\right], e_{s}\right\rangle \partial_{s} \partial_{i}+\frac{1}{4}|v|^{2} \sum_{s=2 m+1}^{2 m+l} \partial_{s}^{2} .
$$

If $f$ is a radial function, then by (1.2)

$$
L f=\left(\frac{\partial^{2}}{\partial r^{2}}+\frac{2 m-1}{r} \frac{\partial}{\partial r}+\frac{1}{4} r^{2} \sum_{s=2 m+1}^{2 m+l} \partial_{s}^{2}\right) f
$$

where $r=|v|$. Applying (4.15) and the equality

$$
r\left(L_{n}^{m-1}\right)^{\prime \prime}(r)+(m-r)\left(L_{n}^{m-1}\right)^{\prime}(r)+n L_{n}^{m-1}(r)=0
$$


[4, vol. 2, p. 188] we obtain that $\mathcal{L}_{n}^{m-1}\left(\frac{1}{2}|w||v|^{2}\right) e^{-i\langle w, z\rangle}$ are eigenfunctions of $L$ with eigenvalues $(2 n+m)|w|$. Let

$$
g_{n}(v, z)=(2 \pi)^{-l-m} \int_{Z} \frac{\hat{P}_{b}(w, n)}{\hat{P}_{a}(w, n)} \mathcal{L}_{n}^{m-1}\left(\frac{1}{2}|w||v|^{2}\right) e^{-i\langle w, z\rangle}|w|^{m} d w .
$$

$g_{n} \in C^{\infty}(N)$ and by (4.13) $\sum_{n=0}^{\infty} g_{n}$ is convergent in $L^{2}(N)$ to $\psi_{a}^{b}$. We have

$$
L^{k} g_{n}(v, z)=(2 \pi)^{-l-m} \int_{Z} \frac{\hat{P}_{b}(w, n)}{\hat{P}_{a}(w, n)}(2 n+m)^{k} \mathcal{L}_{n}^{m-1}\left(\frac{1}{2}|w||v|^{2}\right) e^{-i\langle w, z\rangle}|w|^{m+k} d w
$$

and

$$
\left\|L^{k} g_{n}\right\|_{L^{2}}^{2}=(2 \pi)^{-l-m}\left(\begin{array}{c}
n+m-1 \\
n
\end{array}\right)(2 n+m)^{2 k} \int_{Z}\left|\frac{\hat{P}_{b}(w, n)}{\hat{P}_{a}(w, n)}\right|^{2}|w|^{m+2 k} d w .
$$

Hence by Lemma 4.3

$$
\sum_{n=0}^{\infty}\left\|L^{k} g_{n}\right\|_{L^{2}}^{2}<\infty
$$

This shows that $\psi_{a}^{b}$ belongs to the domain of the closure of $L^{k}$ and so $\psi_{a}^{b}$ is smooth. Since, by [5], for every left-invariant differential operator $\partial$ on $N$ there is a $k$ and $d$ such that

$$
\|\partial f\|_{L^{2}} \leq d\left(\left\|L^{k} f\right\|_{L^{2}}+\|f\|_{L^{2}}\right)
$$

the theorem follows.

\section{REFERENCES}

1. J. Cygan, A tangential convergence for bounded harmonic functions on a rank one symmetric space, Trans. Amer. Math. Soc. 265 (1981), 405-418.

2. E. Damek, A Poisson kernel on type $H$ nilpotent groups, Colloq. Math. (to appear).

3. - The geometry of a semi-direct extension of a type $H$ nilpotent group, Colloq. Math. (to appear).

4. A. Erdélyi, W. Magnus, F. Oberhettinger and F. G. Tricomi, Higher transcendental functiuns, McGraw-Hill, New York, 1953.

5. G. B. Folland, Subelliptic estimates and function spaces on nilpotent Lie groups, Ark. Mat. 13 (1975), 161-207.

6. H. Furstenberg, A Poisson formula for semi-simple Lie groups, Ann. of Math. (2) 77 (1963), 335-386.

7. A. Hulanicki and F. Ricci, A Tauberian theorem and tangential convergence of bounded harmonic functions on balls in $C^{n}$, Invent. Math. 62 (1980), 325-331.

8. A. Kaplan, Fundamental solution for a class of hypoelliptic PDE generated by composition of quadratic forms, Trans. Amer. Math. Soc. 258 (1980), 147-153.

9. A. Korányi, Harmonic functions on symmetric spaces, Symmetric Spaces, Marcel Dekker, New York, 1972.

10. T. Przebinda, A tangential convergence for bounded harmonic functions on a rank one symmetric space (preprint).

11. F. Ricci, Harmonic analysis on generalized Heisenberg groups (preprint).

12. C. Riehm, The automorphism group of a composition of quadratic forms, Trans. Amer. Math. Soc. 269 (1982), 403-414.

Institute of Mathematics, Plac GrunWaldzki 2/4, 50-384 Wroceaw, Poland 